\title{
Linx
}

Revue des linguistes de l'université Paris X Nanterre

68-69 | 2013

Corpus et apprentissage du français

\section{Être et avoir été : l'accord du participe passé par des apprenants de FLE}

\section{Maud Dubois, Alain Kamber et Carine Skupien Dekens}

\section{(2) OpenEdition}

1 Journals

Édition électronique

URL : https://journals.openedition.org/linx/1504

DOI : 10.4000/linx.1504

ISSN : 2118-9692

Éditeur

Presses universitaires de Paris Nanterre

\section{Édition imprimée}

Date de publication : 19 novembre 2013

Pagination : 115-133

ISSN : 0246-8743

\section{Référence électronique}

Maud Dubois, Alain Kamber et Carine Skupien Dekens, « Être et avoir été : l'accord du participe passé par des apprenants de FLE », Linx [En ligne], 68-69 | 2013, mis en ligne le 19 novembre 2015, consulté le 21 septembre 2021. URL : http://journals.openedition.org/linx/1504 ; DOI : https://doi.org/10.4000/ $\operatorname{linx} .1504$ 


\title{
Être et avoir été : l'accord du participe passé par des apprenants de FLE
}

\author{
Maud Dubois, Alain Kamber \& Carine Skupien Dekens, \\ Université de Neuchâtel
}

\section{Introduction}

Tantôt qualifiées d'« incontournable pont aux ânes» (Wilmet, 1999: 7), de «concentré de chausse-trapes » (Béguelin, 2002 : 164) ou de «curiosités orthographiques destinées à donner un subtil plaisir aux seuls happy few » (Leroy \& Leroy, 1995 : 83), les règles d'accord du participe passé en français résistent encore et toujours aux propositions de simplification énoncées par un certain nombre de linguistes (voir récemment Gruaz, 2012; Matthey, 2013) et aux tentatives de réforme officielles ${ }^{1}$. Ces règles constituent un «bastion hautement problématique (Béguelin, 2002 : 171) alors qu'elles ne sont paradoxalement pas entièrement maitrisées par les locuteurs dont le français est la langue maternelle. L'exigence sociale étant importante dans le domaine de l'orthographe, cette situation peut contribuer à maintenir chez certains un «pernicieux sentiment d'insécurité orthographique » (Béguelin, 2002: 164). Décrite pour les locuteurs de français L1, cette réalité est certainement valable aussi pour les apprenants de français L2.

Face aux difficultés et aux incohérences du système, les linguistes et les didacticiens intéressés par la question appellent depuis longtemps de leurs vœux un

${ }^{1}$ Sur la frilosité et la non-efficacité des réformes, voir également Brissaud (1999 : 6-7). Il semblerait néanmoins que certaines propositions de réforme soient perçues plus favorablement par les locuteurs/scripteurs, notamment celle qui viserait à simplifier l'accord du participe passé des verbes pronominaux en permettant dans tous les cas l'accord avec le sujet (Matthey, 2013). 
alignement des règles sur la réalité langagière des locuteurs, ce qui nécessite «de s'informer aussi précisément que possible sur la situation de l'accord du [participe passé] dans le français écrit et parlé aujourd'hui » (Béguelin, 2002 : 177) pour en tirer des conclusions adaptées aux différents types de public, notamment les locuteurs francophones L $2^{2}$. Certaines études citées dans le présent article ont déjà mis en œuvre cette demande en analysant les réalisations orthographiques effectives de scripteurs L1 ou L2. Le développement de la linguistique de corpus permet aujourd'hui de réaliser ce type de recherches à plus large échelle.

Sur la base d'un corpus de textes écrits d'apprenants du français universitaires de niveau B1 et de six langues maternelles différentes comptant 75000 mots, notre étude se propose de procéder à un repérage exhaustif des participes passés (dorénavant PP) dans des formes verbales composées afin d'observer, décrire et expliquer les accords correctement ou incorrectement réalisés. Cette démarche permettra d'esquisser des tendances communes à tous les apprenants ou particulières à chacune des langues représentées et donc de donner des pistes d'explication des réussites ou des erreurs en fonction des L1 des apprenants, dans un but didactique ${ }^{3}$. La présente étude s'inscrit en cela dans les recherches actuelles qui mettent en évidence le rôle des transferts de compétences même partielles - d'une langue à une autre (voir par exemple Galligani \& Bruley, 2014).

Concernant les apprenants de français L2, l'avantage de la recherche sur corpus est double : elle permet d'une part de décrire les pratiques des étudiants par rapport aux règles enseignées ${ }^{4}$ et d'évaluer le taux de réussite dans des textes rédigés librement par des scripteurs ${ }^{5}$; elle permet ainsi, pour le niveau concerné, de mettre au jour les zones de réussite (consciente ou fortuite) et les zones problématiques, ce qui permet in fine d'en tirer des conclusions pertinentes pour l'enseignement / apprentissage du français L2.

Dans cette étude, nous tenons compte exclusivement de critères linguistiques, laissant de côté des éléments socioculturels tels que la culture d'apprentissage des étudiants, leur scolarisation ou leur exposition au français dans leur pays d'origine. Notre recherche se donne pour but de décrire de manière neutre et objective les

${ }^{2}$ La différence entre des locuteurs L1 et L2 apparait clairement dans l'une des études mentionnées dans Matthey (2013).

${ }^{3}$ Les apprenants de niveau B1 ont, à l'écrit, « un bon contrôle grammatical malgré de nettes influences de la langue maternelle » (CECRL). En comparaison, les apprenants de niveau A2 peuvent « utiliser des structures simples correctement mais commet[tent] encore systématiquement des erreurs élémentaires comme, par exemple, la confusion des temps et l'oubli de l'accord. ». Pour une description des compétences orthographiques d'apprenants de niveau B1, voir Luzzati (2011a, 2011b).

${ }^{4}$ Les étudiants B1 pris en considération ici ont reçu un enseignement des règles d'accord du PP non simplifié (et utilisent le même manuel : Skupien Dekens, Kamber \& Dubois, 2011), contrairement à ce qui se fait déjà dans l'enseignement aux apprenants suisses alémaniques, par exemple (voir Matthey, 2013). En effet, nous considérons que pour un public universitaire de niveau B1 qui vise un niveau B2, l'apprentissage des règles en vigueur est indispensable.

${ }^{5}$ L'adverbe «librement » signifie ici que notre recherche ne se base pas sur des dictées ou sur des questionnaires qui testeraient spécifiquement différents cas de figure en matière de règles d'accord du PP, mais sur une tâche de rédaction impliquant à la fois des compétences d'organisation textuelle et des compétences syntaxiques, lexicales et orthographiques. 
difficultés des apprenants dans le cadre d'un enseignement des règles du participe passé non-simplifiées. Elle ne cherche donc à évaluer ni la pertinence des règles en vigueur ni le bien-fondé de propositions de simplification des règles d'accord, ni même l'efficacité de méthodes alternatives d'enseignement de ces règles, simplifiées ou non.

\section{Un corpus et six langues maternelles}

\subsection{Caractéristiques du corpus}

Le corpus utilisé dans cette étude compte un total de 184 textes, qui représentent un peu plus de 75000 mots. Il est homogène sur le plan du niveau de compétence à l'écrit (tous les informateurs retenus présentent un niveau B1) et composé de productions écrites d'apprenants de six L1 différentes: l'anglais, le chinois, l'espagnol, le kurde, le portugais et le russe (Tableau 1).

\begin{tabular}{|c|c|c|c|c|c|c|}
\hline & \multicolumn{2}{|l|}{ Récit } & \multicolumn{2}{|c|}{ Résumé } & \multicolumn{2}{|c|}{ Texte argumentatif } \\
\hline & \multicolumn{6}{|c|}{$\begin{array}{l}\text { Nombre de mots } \\
\text { (entre parenthèses, nombre d'informateurs }=\text { de textes) }\end{array}$} \\
\hline anglais & 916 & (3) & 1121 & (4) & 4174 & (6) \\
\hline chinois & - & $(-)$ & 1804 & (9) & 6165 & (11) \\
\hline espagnol & 2635 & (9) & 4308 & (18) & 19117 & (33) \\
\hline kurde & 493 & (2) & 1956 & $(10)$ & 6213 & (13) \\
\hline portugais & 1345 & (4) & 2594 & (13) & 12904 & (24) \\
\hline russe & 1058 & (5) & 2250 & $(10)$ & 6031 & (10) \\
\hline \multirow[t]{3}{*}{ Total } & 6447 & (23) & 14033 & (64) & 54604 & (97) \\
\hline & \multicolumn{2}{|l|}{$(8,59 \%)$} & \multirow{2}{*}{\multicolumn{2}{|c|}{$(18,69 \%)$}} & \multicolumn{2}{|c|}{$(72,72 \%)$} \\
\hline & & & & & 750 & (184) \\
\hline
\end{tabular}

Tableau 1 : Composition du corpus

Le corpus est composé de trois sous-parties : les résumés $(18,7 \%$ du total de mots) et les textes argumentatifs $(72,7 \%)$ sont des travaux obligatoires rédigés hors classe par les étudiants, alors que les récits $(8,6 \%)$ ont été écrits en classe sans recours au dictionnaire ni au vérificateur d'orthographe ${ }^{6}$. S'il ne peut être considéré comme équilibré7, ce corpus contient néanmoins des textes qui obéissent à des règles de rédaction très diverses, créés dans des contextes de production différents, et offre donc un terrain d'exploration relativement représentatif des compétences générales de nos informateurs à l'écrit.

Le corpus a été annoté au moyen du logiciel TreeTagger ${ }^{8}$. Le choix de ce logiciel est motivé par notre volonté de procéder à une analyse exhaustive des textes

\footnotetext{
${ }^{6}$ Pour cette catégorie de textes, nous avons demandé aux étudiants de raconter leurs premières impressions à leur arrivée en Suisse, les forçant ainsi à utiliser les temps du passé, et plus particulièrement le passé composé.

${ }^{7}$ Sur l'impossibilité fondamentale de constituer des corpus équilibrés, voir Péry-Woodley (1995: 218) et Teubert (1998:131).

${ }^{8}$ Développé par Helmut Schmid (Stuttgart) et utilisable pour une quinzaine de langues, TreeTagger permet d'attribuer à chaque mot d'un texte une catégorie grammaticale. http ://www.ims.unistuttgart.de/projekte/corplex/Tree'Tagger/.
} 
sans annotation préalable des erreurs, ceci pour éviter de biaiser les résultats?. L'étiquetage sur les parties du discours (classes de mots) et les informations de lemmatisation nous ont permis dans un premier temps de marquer tous les PP, puis d'isoler tous les temps composés.

\subsection{L'accord du participe passé : une spécificité française}

Dans cette étude, quatre cas de figure impliquant l'accord ou non du PP seront analysés :

- temps composés conjugués avec l'auxiliaire avoir;

- temps composés conjugués avec l'auxiliaire être;

- temps composés des verbes pronominaux ;

- formes verbales au passif ${ }^{10}$.

Outre ces quatre catégories, nous tiendrons compte des L1 des apprenants. On peut en effet aisément imaginer que le système d'accord du PP du français pose des problèmes à un étudiant dont la langue maternelle présente un système différent dans les domaines des accords (genre et/ou nombre) et de la morphologie verbale (plus particulièrement des temps du passé ${ }^{11}$.

Le Tableau 2 permet de voir comment chacun des systèmes linguistiques des L1 représentées fonctionne du point de vue de la morphologie verbale (des temps composés et de leurs éventuels équivalents) et - le cas échéant - de l'accord du $\mathrm{PP}^{12}$. Ce tableau appelle quelques commentaires :

- l'anglais ne connait que l'auxiliaire to have ('avoir') pour les temps composés, alors que le passif se construit avec l'auxiliaire to be ('être'). Le PP ne s'accorde jamais ;

- le chinois ne connait pas de formes composées, la valeur perfective - comme les relations temporelles en général - s'y exprime par l'adjonction d'éléments à valeur adverbiale. Le passif en tant que tel n'existe pas: on peut exprimer cette valeur par l'ajout devant le verbe d'un mot tel que bèi, ái ou shòu, selon le contexte, ou par l'inversion des mots ;

- en espagnol, l'accord du PP - toujours utilisé avec haber ('avoir') dans les temps composés, également pour les verbes pronominaux - ne pose aucun problème : il ne

\footnotetext{
${ }^{9}$ Sur les corpus d'apprenants sans annotation des erreurs, voir Rastelli \& Frontini (2008), et surtout Rastelli (2009), qui affirme: «[...] it is better to work with "virtual categories" rather than with errors. » Pour des pratiques incluant l'annotation des erreurs, voir notamment Granger (2003), Lüdeling et al. (2005) ainsi que Albert et al. (2010).

${ }^{10}$ Cette quatrième catégorie vient s'ajouter à celles communément retenues dans les études d'analyse des erreurs d'accord du PP, et notamment dans le catalogue de Catach, Duprez \& Legris (1980: 14).

${ }^{11}$ Sur le rôle exercé par le système de référence d'un étudiant dans les difficultés qu'il va avoir pour maitriser les règles de la langue cible, voir Nickel \& Nehls (1982), Kattenbusch (1993) et plus récemment Granfeldt \& Ågren (2014) pour les accords sujet-verbe d'apprenants du français suédophones.

${ }^{12}$ Nous tenons à remercier sincèrement les personnes qui nous ont renseignés sur les langues maternelles des apprenants : Eunice De Jesus Mateiro da Silva, Juliana Duarte Martins, Rosa et Kemal Topalak, Yiqi Liu, Lise-Marie Moser, Natacha Reynaud Oudot, Irina Solomatina Tissot, Cyril Tissot et Michelle Vauthier.
} 
s'accorde jamais. Le passif est formé avec les auxiliaires ser ou estar ('être'), dans ce cas le PP s'accorde en genre et en nombre avec le sujet ;

- le kurde connait de fortes variations dialectales. Nous nous appuyons ici sur la description morphosyntaxique de la variété du nord, le kurmandji (voir Bedir Khan \& Lescot, 1970);

- si l'équivalent portugais du passé composé est un temps simple, le preterito perfeito, les temps composés existent dans cette langue et se forment en principe avec l'auxiliaire ter ('avoir') - haver étant en principe considéré comme littéraire - avec un PP invariable. Ceci est valable également pour les verbes pronominaux. Comme en espagnol, le passif est formé avec les auxiliaires ser ou estar ('être') avec accord en genre et en nombre du PP avec le sujet ;

- le russe ne connait pas de formes composées, et ne comporte donc ni auxiliaire ni PP ; le perfectif est marqué par des préfixes ou des allomorphes. Les verbes pronominaux existent et se forment par suffixation; il en va de même du passif.

\begin{tabular}{|c|c|c|c|c|c|c|c|c|c|c|c|c|}
\hline & & \multicolumn{4}{|c|}{ Auxiliaire avoir } & \multicolumn{2}{|c|}{$\begin{array}{l}\text { Auxiliaire } \\
\text { ettre }\end{array}$} & \multicolumn{3}{|c|}{$\begin{array}{l}\text { Verbes } \\
\text { pronominaux }\end{array}$} & \multicolumn{2}{|c|}{ Passif } \\
\hline & 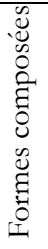 & 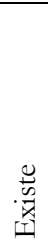 &  & 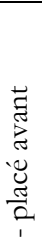 & 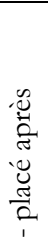 & 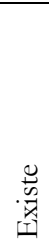 & 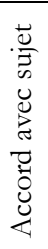 & 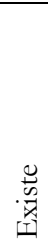 & 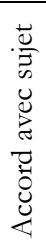 & 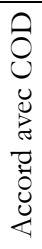 & 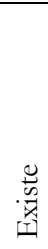 & 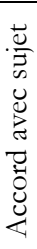 \\
\hline français & + & + & + & + & - & + & + & + & + & + & + & + \\
\hline anglais & + & + & - & - & - & - & - & + & - & - & + & - \\
\hline chinois & - & - & - & - & - & - & - & - & - & - & - & - \\
\hline espagnol & + & + & - & - & - & - & - & + & - & - & + & + \\
\hline kurde & + & - & - & - & - & + & - & + & - & - & + & - \\
\hline portugais & + & + & - & - & - & - & - & + & - & - & + & + \\
\hline russe & - & - & - & - & - & - & - & + & - & - & + & - \\
\hline
\end{tabular}

Tableau 2 : Caractéristiques morphologiques des langues sources impliquées

\section{Analyse empirique}

Sur l'ensemble du corpus, les PP ne représentent qu'1,76\% du total des mots. Si l'accord du PP est certainement un chapitre important sur le plan théorique, et s'il occupe d'ailleurs traditionnellement une place de choix dans l'enseignement de l'écrit, il n'en constitue pas moins un phénomène relativement marginal sur le strict plan quantitatif dans le corpus analysé. Au total, 1321 cas ont été observés (Tableau 3). De façon générale, on constate sans surprise une prédominance des formes composées avec l'auxiliaire avoir (756 occurrences, soit $57,22 \%$ du total), cas majoritaire aussi bien dans les types que dans les tokens en français. Les formes composées avec être en revanche sont largement moins représentées (148 occurrences, soit 11,20\% du total), alors que les verbes pronominaux (67) ne représentent que 5,07\% du total des formes verbales composées. Enfin, on relèvera le nombre important de formes verbales au passif (350 occurrences, soit $26,50 \%$ du total). 


\begin{tabular}{|l|r|r|r|r|r|r|r|}
\hline Catégorie & \multicolumn{3}{|l|}{ Formes correctes } & \multicolumn{3}{|l|}{ Formes incorrectes } & \multirow{2}{*}{ Total } \\
\cline { 3 - 7 } & \multicolumn{2}{|l|}{} & \multicolumn{2}{|l|}{ Auxiliaire } & \multicolumn{2}{l|}{ Participe passé } & \\
\hline Auxiliaire avoir & 693 & $(91,67 \%)$ & 13 & $(1,72 \%)$ & 50 & $(6,61 \%)$ & 756 \\
\hline Auxiliaire être & 99 & $(66,89 \%)$ & 24 & $(16,22 \%)$ & 25 & $(16,89 \%)$ & 148 \\
\hline Pronominaux & 54 & $(80,60 \%)$ & 4 & $(5,97 \%)$ & 9 & $(13,43 \%)$ & 67 \\
\hline Passif & 306 & $(87,43 \%)$ & 2 & $(0,57 \%)$ & 42 & $(12,00 \%)$ & 350 \\
\hline Total & $\mathbf{1 1 5 2}$ & $(87,21 \%)$ & $\mathbf{4 3}$ & $(3,26 \%)$ & $\mathbf{1 2 6}$ & $(9,54 \%)$ & $\mathbf{1 3 2 1}$ \\
\hline
\end{tabular}

Tableau 3 : Total des PP dans le corpus (entre parenthèses,

les pourcentages de formes correctes / incorrectes ${ }^{13}$ par type de forme composée)

En ce qui concerne la proportion des formes correctes / incorrectes, toutes catégories confondues, on observe que le nombre de formes correctes de PP est très important (1152, soit $87,2 \%$ du total des formes analysées). Les erreurs observées (169 ou 12.8\% du total) se répartissent en fonction de deux critères : mauvais choix de l'auxiliaire (un quart des erreurs) et accord fautif du participe (trois quarts des erreurs, mais moins de $10 \%$ du total des formes analysées).

Si on s'intéresse aux différentes catégories de formes verbales, on constate que les formes composées avec avoir présentent un taux de formes correctes supérieur à la moyenne $(91,67 \%$ pour $87,21 \%)$. En revanche, les formes composées avec l'auxiliaire être ont le taux largement le plus bas $(66,89 \%)$, alors que pour le passif, où le PP doit aussi toujours s'accorder, le taux de réussite est dans la moyenne (87,43\%).

En ce qui concerne les erreurs dues au choix de l'auxiliaire, les formes avec avoir présentent un taux inférieur à la moyenne $(1,72 \%$ pour $3,26 \%)$ et à l'inverse, celles avec être un taux nettement supérieur $(16,22 \%)$. Les formes passives, en revanche, ne posent pour ainsi dire aucun problème de choix de l'auxiliaire.

La deuxième source d'erreurs, l'accord du PP, s'avère également la plus présente avec l'auxiliaire être (où le choix de l'auxiliaire et l'accord du PP représentent quasiment le même nombre de fautes), alors que les formes avec avoir présentent un taux largement plus bas que les autres catégories. Enfin, les formes passives, qui ne comportent pour ainsi dire pas d'erreurs sur le choix de l'auxiliaire, en contiennent sur l'accord des PP.

La répartition des formes correctes / incorrectes en fonction des L1 des apprenants est présentée dans le Tableau 4.

\footnotetext{
${ }^{13}$ Sont considérées comme correctes les formes avec le bon auxiliaire et un PP morphologiquement juste et graphiquement bien accordé.
} 
Être et avoir été : l'accord du participe passé par des apprenants de FLE

\begin{tabular}{|l|r|r|r|}
\hline \multirow{2}{*}{ Langues } & \multicolumn{2}{|l|}{$\begin{array}{l}\text { Formes } \\
\text { correctes }\end{array}$} & \multicolumn{2}{|c|}{ Formes incorrectes } \\
\cline { 3 - 4 } & 80,87 & auxiliaire & \multicolumn{1}{c|}{ PP } \\
\hline anglais & 93,22 & 4,35 & 14,78 \\
\hline chinois & 85,49 & 1,69 & 5,08 \\
\hline espagnol & 89,35 & 3,30 & 11,21 \\
\hline kurde & 85,95 & 4,14 & 6,51 \\
\hline portugais & 92,12 & 3,68 & 10,37 \\
\hline russe & 87,21 & 1,82 & 6,06 \\
\hline moyenne & 3,26 & 9,54 \\
\hline
\end{tabular}

Tableau 4 : Proportion des PP corrects et incorrects selon les L1

On constate de façon générale que la proportion de formes composées correctement réalisées est de $87,21 \%$ toutes langues confondues. Cependant, d'importantes disparités sont à relever. Ainsi, les anglophones se situent en-dessous de la moyenne, avec un taux de réussite de $80,87 \%$. A l'inverse, les kurdophones $(89,35 \%)$, et surtout les sinophones et les russophones présentent des taux supérieurs à la moyenne $(93,22 \%$ et $92,12 \%)$; ce dernier fait est d'autant plus remarquable que le chinois et le russe ne connaissent pas, dans leur morphosyntaxe, de formes composées. On pourrait ici formuler l'hypothèse que l'absence de temps composés dans ces deux langues oblige les apprenants à intégrer complètement un système différent du leur et rend impossible la formation de calques erronés. Quant au kurde, s'il connait des formes composées pour certains temps et modes, l'équivalent du passé composé français est un temps simple (prétérit).

En ce qui concerne le choix de l'auxiliaire, les deux groupes qui présentent le plus d'erreurs sont les anglophones et les kurdophones. Dans ces deux langues, il n'y a qu'un auxiliaire pour les temps composés : to have ('avoir') pour l'anglais et bûn ('être') pour le kurde. Hispanophones et lusophones, qui connaissent eux aussi un seul auxiliaire (haber et ter / aver, 'avoir') se situent grosso modo dans la moyenne, alors que sinophones et russophones ne commettent que très peu d'erreurs.

Pour les fautes d'accord du PP, on constate une grande proportion d'erreurs chez les anglophones (14,78\% pour un taux moyen de 9,54\%), dont le PP est toujours invariable. Les hispanophones et les lusophones, dont le PP ne varie qu'au passif, présentent eux aussi un taux d'erreurs supérieur à la moyenne.

\subsection{Les participes passés avec avoir}

Cette première partie de l'analyse s'intéressera à la catégorie la plus représentée dans notre corpus, le PP des verbes avec l'auxiliaire avoir. Le Tableau 5 présente, sous forme de pourcentages, les résultats obtenus en fonction de la L1 des informateurs :

\begin{tabular}{|l|l|l|r|r|}
\hline Catégorie & Langues & \multicolumn{2}{l|}{$\begin{array}{l}\text { Formes } \\
\text { correctes }\end{array}$} & \multicolumn{2}{l|}{ Formes incorrectes } \\
\cline { 3 - 5 } & & 89,23 & 3,08 & 7,69 \\
\hline \multirow{4}{*}{ AVOIR } & anglais & 93,85 & 1,54 & 4,62 \\
\cline { 2 - 5 } & chinois & 87,80 & 1,18 & 11,02 \\
\cline { 2 - 5 } & espagnol & 93,52 & 2,78 & 3,70 \\
\cline { 2 - 5 } & kurde & 94,58 & 1,81 & 3,61 \\
\cline { 2 - 5 } & portugais & 94,90 & 1,02 & 4,08 \\
\cline { 2 - 5 } & russe & 91,67 & 1,72 & 6,61 \\
\hline
\end{tabular}

Tableau 5 : Pourcentage de réussite des PP avec avoir dans le corpus 


\subsubsection{Formes correctes}

Ainsi que nous l'avons vu plus haut, l'accord du participe avec l'auxiliaire avoir présente le taux de réussite le plus élevé. Ce résultat pourrait indiquer qu’à un niveau $\mathrm{B} 1$ les apprenants maitrisent dans une large mesure les règles complexes de l'accord du PP avec avoir, dont l'application nécessite en effet des compétences d'analyse grammaticale et syntaxique certaines: outre le repérage d'un PP à accorder, il s'agit d'identifier la présence ou non d'un COD, ainsi que sa position (après ou avant le verbe $)^{14}$.

Si l'on analyse plus précisément les formes verbales correctement réalisées, ce constat optimiste doit être nuancé. En effet, sur les 693 formes sans erreur, 637 ne nécessitent pas d'accord du PP : 460 contiennent un COD placé après le verbe (ex. 1) et 177 n'ont pas de COD (2) :

1. Il faut savoir que grâce à nos parents on a eu l'opportunité d'exister, d'être ce qu'on est maintenant, grâce à eux on a vécu une grande série d'événements [...].

2. Tous les deux ont voyagé beaucoup [...].

Dans le premier cas comme dans le second, nous ne savons pas si les étudiants ont analysé leur phrase pour déterminer si oui ou non il y avait un COD et quelle était sa place ou s'ils ont appliqué une règle simplifiée du type «avec l'auxiliaire avoir, pas d'accord».

Seules 56 formes verbales avec un COD apparaissant avant le verbe ont été correctement orthographiées. Parmi celles-ci, il faut encore préciser que 31 concernent un COD au masculin singulier, ce qui implique que le PP reste invarié (3). Par ailleurs, sur les 9 PP corrects qui devaient être accordés au masculin pluriel, 3 sont des participes qui ont un $-\mathrm{s}$ au singulier (4). Restent donc 22 formes verbales pour lesquelles nous pouvons penser que les étudiants ont consciemment et correctement appliqué la règle $(5 \text { et } 6)^{15}$ :

3. Cependant le voyage que j'ai entrepris avec un ami direction Istanbul, en passant par l'Italie et la Grèce, fut celui me fit sortir des sentiers battus.

4. [L]es catastrophes naturelles des dernières années nous ont surpris [...].

5. Pour préciser mon propos, je prendrai l'exemple suivant: Claire, Zoé et Matteo trois personnes que j'ai connues.

6. [Q]uand elle devra prendre une décision, elle pourra appeler à son aide les critères de l'habitude et de la routine qu'elle a déjà établis pour organiser son quotidien.

\footnotetext{
${ }^{14}$ Sur les limites de «cette notion, discutée et discutable » qu'est le COD, voir Béguelin (2002:165) et Berrendonner (1983: 43-45).

${ }^{15}$ Dans quelques cas où le COD est du même genre et nombre que le sujet, on peut néanmoins voir en observant le cotexte que l'accord a plutôt été fait avec le sujet, comme dans l'exemple suivant, dont l'auteur est une femme : *Je l'avais trouvée [la Suisse] de nouveau très belle comme avant [...] j'ai visitée plusieurs endroits comme les châteaux, les lacs, etc.
} 
Le corpus utilisé ici tend ainsi à montrer que les étudiants produisent spontanément nettement plus d'énoncés dans lesquels le COD est placé après le verbe $(62,83 \%)$ ou sans COD $(24,34 \%)$ que d'énoncés dans lesquels le COD est placé avant $(10,58 \%)^{16}$. Ceci pourrait nous faire penser qu'ils sous-utilisent les relatives et l'usage des pronoms, avec comme corollaire une majorité de cas où le PP reste invariable. Cette tendance apparait de manière très nette pour toutes les langues mais elle est particulièrement visible chez les anglophones dont les PP corrects avec un COD placé avant représentent environ 3,5\% seulement des formes correctes (alors que la moyenne est de $8 \%$ ). A l'inverse, les sinophones produisent près de $11,5 \%$ de leurs PP corrects avec cette syntaxe. L'étude menée par Leroy \& Leroy (1995) indique toutefois que dans les textes écrits de francophones adultes, la proportion de PP qui restent invariables ou invariés est très élevée $(85 \%)$, avec comme conséquence le fait que la règle simplifiée selon laquelle le PP ne s'accorde pas avec avoir est opérante environ 9 fois sur 10 .

S'il semble évident que les résultats obtenus auraient été bien différents dans le cas où nous aurions testé l'application des règles au moyen d'une dictée truffée de PP apparaissant dans les différentes configurations possibles et par là même concentrée en difficultés, il n'en demeure pas moins qu'au vu de la syntaxe qu'ils privilégient spontanément, les étudiants de niveau B1 font très peu de fautes dans l'accord du PP avec l'auxiliaire avoir. Cela signifie également que, dans une grande majorité de cas, ils ne surgénéralisent pas avec avoir la règle d'accord avec le sujet qui prévaut dans le cas du PP avec l'auxiliaire être.

\subsubsection{Formes incorrectes}

Si l'on considère les erreurs commises et les différences saillantes entre scripteurs en fonction de leur langue maternelle, un premier constat concerne le fait que les anglophones et, de manière encore plus marquée, les hispanophones sont les étudiants qui ont les taux les plus élevés de formes composées avec avoir mal accordées : environ $7,7 \%$ pour les premiers et $11 \%$ pour les seconds, alors que la moyenne est de $6,6 \%$ et que les quatre autres groupes se situent entre 3,5 et 4,5\% d'erreurs. Ce résultat n'est pas surprenant dans la mesure où en espagnol et en anglais le PP est invariable ${ }^{17}$.

Concernant les différents types d'erreurs sur l'accord du PP avec l'auxiliaire avoir, le plus grand nombre de formes mal orthographiées est, de manière attendue, celles où le COD est placé avant le verbe. Très peu d'erreurs sont présentes avec un COD qui suit le PP $(3,2 \%)$ ou sans COD $(3,8 \%)$. Dans ces cas où le PP doit rester invariable, toutes L1 confondues, l'accord est majoritairement effectué avec le sujet (7 et 8$)$ :

7. *Je pense qu'ils ont oubliés le rôle de la famille.

\footnotetext{
${ }^{16}$ Les $2,7 \%$ restants sont ceux où l'on observe un choix fautif de l'auxiliaire.

${ }^{17}$ Sans que l'on puisse y trouver une raison objective, il faut toutefois remarquer que les étudiants lusophones - eux aussi confrontés à cette différence importante entre le français et leur L1 réussissent à mieux appliquer les règles d'accord.
} 
8. *Tous les matins j'ai travaillée avec mes collègues [...].

Lorsque le COD est placé avant le verbe, le taux de PP mal accordés augmente notablement puisqu'il passe à 27,3\%. L'analyse détaillée des erreurs commises dans cette configuration montre, de manière attendue là aussi, qu'elles concernent essentiellement des syntagmes verbaux dans lesquels le COD n'est pas au masculin singulier. Si le pourcentage d'erreur pour ce dernier cas est de $6,1 \%$, il passe à $54,2 \%$ pour un COD féminin singulier, 30,8\% lorsqu'il est masculin pluriel et 50\% s'il est féminin pluriel. Bien que les chiffres pris en compte ici soient trop peu représentatifs pour pouvoir avoir valeur de preuve ( 24 participes mal accordés avec COD avant $)^{18}$, ils semblent néanmoins montrer que la variable du genre est celle qui pose le plus de problème aux étudiants pour l'accord du PP, bien que la catégorie du genre existe dans 4 des 6 L1 représentées dans le corpus ${ }^{19}$. Cette tendance sera confirmée par les autres catégories de verbes examinées plus bas.

Les cas où les étudiants accordent mal les PP avec un COD placé avant sont quelque peu différents de ceux où le PP est invariable: tandis que ce dernier type d'erreur est dû à une application à mauvais escient de l'accord avec le sujet, dans la majorité des cas où un PP est mal accordé avec un COD placé avant (19 sur 24), le PP n'est pas accordé du tout (9 et 10). Seuls 4 cas sur 24 présentent un accord avec le sujet (11 et 12). Dans la mesure où ces deux types d'erreurs sont en grande partie celles des hispanophones et, pour une moindre part, des lusophones (dont les sous-corpus sont les plus importants en nombre de mots), ce résultat confirme la difficulté rencontrée par les premiers dans l'accord du PP :

9. *Je me souviens que la premier neige que j’ai touché j’ai la mis directement à ma bouche.

10. *Ainsi, elles revendiquaient la différance salariale, et elles l'on obtenu.

11. *Quand j’ai montée à la voiture, une des premières choses qui m'ont impressionnées était le trafic [...].

12. *[E]lle a parlé même des disputes qu’il y a eu, des claques qu'elle a ramassée [...].

L'analyse des erreurs commises dans l'accord du PP avec l'auxiliaire avoir montre que parmi les règles apprises dans le cadre de l'accord du PP, l'accord avec le sujet et l'invariabilité sont les deux options privilégiées par les étudiants ${ }^{20}$. Aucun cas où un apprenant choisirait comme mauvaise solution l'accord avec le COD n'apparait

\footnotetext{
18 Ces 24 occurrences ne représentent que 3,17\% du total des formes composées avec l'auxiliaire avoir et constituent donc une partie somme toute minime.

19 Seuls l'anglais et le chinois ne connaissent pas cette catégorie. Voir Dubois, Kamber \& Skupien Dekens (2014).

${ }^{20}$ Ces résultats recoupent largement ceux auxquels aboutit l'étude que Gervaix (1995) a menée avec des jeunes francophones de $2^{\mathrm{e}}$ et $3^{\mathrm{e}}$ années secondaires : les erreurs qui concernent l'accord avec l'auxiliaire avoir consistent soit à accorder avec le sujet, soit, plus fréquemment, à laisser le PP invarié. Voir également Brissaud (1999 : 14).
} 
dans le corpus ${ }^{21}$. Ce constat rejoint les observations faites depuis un certain nombre d'années déjà sur la tendance à l'invariabilité du participe avec l'auxiliaire avoir et un COD placé avant chez les francophones, à l'oral ou à l'écrit ${ }^{22}$.

\subsection{Les participes passés avec être}

Contrairement à celle qui préside à l'accord des PP conjugués avec l'auxiliaire avoir, la règle d'accord des verbes avec être ne pose théoriquement pas de problème : le PP s'accorde en genre et en nombre avec le sujet. Les productions des apprenants nous montrent (Tableau 6) cependant que l'application de cette règle ne va pas sans problème.

\begin{tabular}{|l|l|r|r|r|}
\hline Catégorie & Langues & \multicolumn{2}{|l|}{$\begin{array}{l}\text { Formes } \\
\text { correctes }\end{array}$} & \multicolumn{2}{|l|}{ Formes incorrectes } \\
\cline { 3 - 5 } & & Auxiliaire & \multicolumn{1}{c|}{ Participe passé } \\
\hline \multirow{E}{*}{ ETRE } & anglais & 63,16 & 15,79 & 21,05 \\
\cline { 2 - 5 } & chinois & 88,89 & 11,11 & 0,00 \\
\cline { 2 - 5 } & espagnol & 69,09 & 18,18 & 12,73 \\
\cline { 2 - 5 } & kurde & 86,67 & 0,00 & 13,33 \\
\cline { 2 - 5 } & portugais & 47,22 & 22,22 & 30,56 \\
\cline { 2 - 5 } & russe & 78,57 & 14,29 & 7,14 \\
\hline Moyenne & & 66,89 & 16,22 & 16,89 \\
\hline
\end{tabular}

Tableau 6 : Pourcentage de réussite des PP avec être dans le corpus

\subsubsection{Formes correctes}

Sur les 148 formes verbales composées avec l'auxiliaire être recensées dans notre corpus, 99 sont correctement réalisées, soit 66,89\%. Parmi ces 99 formes correctes, 40 sont non-marquées (masc. sing.) (ex. 13), 39 au fém. sing. (14), 18 au masc. plur. (15) et 2 seulement au fém. plur. (16) :

\section{Enfin le grand jour est arrivé !}

14. Je suis arrivée en suisse de l'Angleterre au debut du septembre 2010 pour faire l'exchange (stage) erasmus entre l'université de Kent (mon université) et l'université de Neuchâtel.

15. Dans ce sens, je connais quelques amis qui sont restés célibataires par choix et sont bien dans leur peau $[\ldots]$.

16. Quelques semaines après les feuilles des arbres sont tombées et moi je pensait : "Combien de temps faut-il pour devenir un vrai arbre avec de feuilles verts?»

Les proportions des formes correctes / incorrectes selon le genre et le nombre s'avèrent intéressantes, même si le nombre modeste d'occurrences oblige à une certaine prudence. On constate ainsi que le taux de réussite pour les formes masculines (masc. sing. : 85,1\% ; masc. plur. : 62\%) est plus élevé que pour les formes féminines

${ }^{21}$ Dans un très petit nombre de cas, il est possible de se demander si l'étudiant(e) a fait l'accord avec un COD placé après (*j'ai vue la neige, *cette dame a découverte une plaque commémorative) mais le je étant féminin dans le premier cas cité et la majorité d'accord avec le sujet dans les cas où le PP doit rester invariable (ici COD après) font pencher pour l'accord avec le sujet.

22 Voir notamment Frei (1929 : 56-57), Catach (1989 : 289), Wilmet (1999 : 24) et Gruaz (2012 : 49-52). 
(fém. sing. : 56,5\% ; fém. plur. : 40\%). Il semblerait donc que c'est ici aussi le critère de genre qui simplifie ou complique ici la tâche des apprenants ${ }^{23}$.

\subsubsection{Formes incorrectes}

Ces considérations ne doivent cependant pas occulter le fait que les formes verbales avec être présentent, largement, le taux de réussite le plus bas parmi les quatre catégories analysées dans cet article (66,89\% pour un taux moyen de 87,21\% pour l'ensemble du corpus). Les erreurs enregistrées se répartissent de façon quasiment égale entre l'utilisation du mauvais auxiliaire (24 occurrences, 16,22\%) et les PP mal accordés (25 occurrences, 16,89\%).

Le pourcentage très élevé d'erreurs dans le choix de l'auxiliaire $(16,22 \%$, alors que le taux pour l'ensemble des formes composées de notre corpus est de 3,26\% seulement) s'explique certainement principalement par l'absence de choix dans les différentes L1 des apprenants : l'anglais, l'espagnol et le portugais ne connaissent que l'auxiliaire avoir, le chinois et le russe n'ont pas de formes composées; le kurde, en revanche, ne connait qu'un auxiliaire être, ce qui pourrait éventuellement expliquer l'absence d'erreurs de ce groupe d'apprenants ${ }^{24}$. L'exemple 17, d'une étudiante anglophone, est particulièrement intéressant :

17. *J'ai commencé a voyagé en dehors Neuchâtel apès; j’ai sorti en boit a genève, Lausanne, j’ai fait la tour de Bern, Zurich et je suis même allée faire du ski.

Dans cet extrait, nous observons en effet la présence simultanée d'une forme verbale correcte, avec auxiliaire être et accord en genre et en nombre du PP (je suis allée) et d'une autre qui contient l'auxiliaire avoir (j'ai sorti en boit). Cette phrase montre que cette apprenante est capable d'utiliser correctement une forme fréquente et sans doute apprise très tôt, mais qu'elle ne peut pas encore, à son niveau B1, produire un énoncé correct à chaque fois.

En ce qui concerne le deuxième type d'erreurs, l'accord fautif du PP, on constate une large prédominance des cas sans aucun accord (22 occurrences sur 25) :

18. *Je voyais les jours gris, beaucoup de nuages, de la pluie et même la neige est venu !

Dans trois cas seulement l'erreur est due à un accord fautif :

19. *Il y'a des femmes et des hommes qui n'ont pas des enfants et leurs parent sont mortes et ils sont des enfants unique et sa leur dérangent pas leur vie professionnel est la chose la plus important pour eux.

La conclusion qui s'impose ici est donc que, si le principe de l'accord du PP avec le sujet est a priori très simple, en revanche sa mise en application représente un réel problème pour les apprenants. En effet, même s'ils ne maitrisent pas forcément

\footnotetext{
${ }^{23}$ Pour l'accord de l'adjectif épithète, à l'inverse, Dubois, Kamber \& Skupien Dekens (2014) ont démontré que c'est le nombre - plus particulièrement le pluriel - qui constitue le problème le plus difficile à résoudre. Voir également Fayol (2007).

24 On verra cependant plus bas que les kurdophones commettent des erreurs dans le choix de l'auxiliaire des verbes pronominaux.
} 
moins bien la règle de l'accord avec être qu'avec avoir, les erreurs sont par nature plus visibles ici (rappelons qu'avec avoir, on a une très large majorité de cas où le PP est invariable, car sans COD ou avec un COD placé après).

\subsection{Les participes passés des verbes pronominaux}

L'accord du PP des verbes pronominaux représente sans conteste, sur le plan des règles, un cas complexe même pour des scripteurs francophones ${ }^{25}$. Le Tableau 7 nous montre cependant que le taux de PP correctement accordés est largement supérieur à celui des verbes avec être :

\begin{tabular}{|l|l|r|r|r|}
\hline Catégorie & Langues & \multicolumn{2}{|l|}{$\begin{array}{l}\text { Formes } \\
\text { correctes }\end{array}$} & \multicolumn{2}{l|}{ Formes incorrectes } \\
\cline { 3 - 5 } & & Auxiliaire & \multicolumn{1}{c|}{ Participe passé } \\
\hline \multirow{3}{*}{ Pronominaux } & anglais & 75,00 & 0,00 & 25,00 \\
\cline { 2 - 5 } & chinois & 100,00 & 0,00 & 0,00 \\
\cline { 2 - 5 } & espagnol & 62,50 & 12,50 & 25,00 \\
\cline { 2 - 5 } & kurde & 77,78 & 22,22 & 12,50 \\
\cline { 2 - 5 } & portugais & 87,50 & 0,00 & 11,11 \\
\cline { 2 - 5 } & russe & 88,89 & 0,00 & 13,43 \\
\hline
\end{tabular}

Tableau 7 : Pourcentage de réussite des PP des verbes pronominaux dans le corpus

\subsubsection{Formes correctes}

Même si le nombre restreint de cas apparaissant dans le corpus (67) ne permet guère de tirer des conclusions véritablement significatives, on peut néanmoins observer quelques tendances. Ainsi, la règle qui veut que tout verbe pronominal du français se conjugue avec l'auxiliaire être semble plus ou moins acquise, même par les locuteurs de langues dans lesquelles ce n'est pas le cas. Les seules exceptions (au nombre de 4) sont le fait d'apprenants hispanophones (ex. 20) et - plus étonnant, cette langue ne possédant pas d'auxiliaire avoir - kurdophones :

20. Cette question permet de s'interroger si vraiment on lui donne à la famille l'importance et l'attention qu'elle mérite, pas seulement pour s'avoir occupé de nous quand on était petit, pour nous aider à passer les obstacles qui se nous présentent chaque jour ou simplement pour le fait d'être les personnes qui nous aiment plus et qui s'inquiètent quand on ne va pas bien.

Si l'on s'intéresse aux PP correctement accordés (54 occurrences), on constate que le corpus ne présente aucun cas d'accord avec un COD placé avant autre que le pronom réfléchi. Par ailleurs, il contient un seul exemple de PP invariable :

21. [...] je me suis rendu compte que je vivais une vie qui me plaisais, que j'étais heureuse même si je pensais avoir une vie parfois ennuyante.

L'utilisation correcte de cette locution à verbe support (dans laquelle on peut considérer compte comme un COD placé après) est d'autant plus remarquable que le

25 Pour une tentative d'établir une procédure d'analyse opérationnelle, voir par exemple Skupien Dekens, Kamber \& Dubois (2011: 58). 
sujet est féminin, renforçant le risque d'un accord au féminin. On peut dès lors légitimement se demander s'il s'agit du produit d'une véritable analyse grammaticale ou du fruit du hasard.

Les 53 autres cas nécessitent un accord avec le sujet. Dans ce dernier groupe, 21 formes sont non-marquées (masc. sing.) (22), 20 au masc. plur. (23), 10 au fém. sing. (24) et 2 seulement au fém. plur. (25), ce qui correspond globalement aux proportions notées pour les verbes composés avec l'auxiliaire être :

22. Cependant quand il s'est marié et a eu des enfants, et il a changé complétement sa vision de la famille.

23. Et ce jour-là, nous nous sommes installés dans une petite maison qui est située à Saint Blaise, tout près du moulin et du Temple [...].

24. [...] d'une certain façon elle augmente son auto-estime parce qu'elle s'est convaincue d'être capable de régir son monde ou une partie de lui.

25. Quatre ans après une réévaluation, elles se sont retrouvées en égalité.

De façon générale, on observe que ces extraits, outre l'accord correct du PP, présentent une certaine richesse de syntaxe et de lexique, et on pourrait de prime abord imaginer que leurs auteurs ont bénéficié d'une aide extérieure au moment de la rédaction. Cette hypothèse peut toutefois clairement être écartée pour l'exemple (23), rédigé en classe sans dictionnaire ni correcteur orthographique et sous contrainte de temps par une apprenante anglophone. Enfin, il est remarquable que les deux seuls essais impliquant le féminin pluriel recensés dans le corpus soient réussis.

\subsubsection{Formes incorrectes}

Si l'on s'intéresse maintenant aux PP mal accordés, on peut distinguer deux sources d'erreurs : l'absence d'accord (6 occurrences sur 9$)$ et l'accord fautif avec le sujet (3 occurrences). Dans le premier cas, l'apprenante n'a pas conscience d'avoir affaire à un verbe transitif direct et à un pronom réfléchi COD :

26. *Apés quelque semaine je [fém.] me suis habitué a la vie suisse, quelque chose que j’ai ne pouvais jamais imaginer au début du semestre.

Dans le second, en revanche, c'est le caractère transitif indirect du verbe qui a échappé à l'apprenante, et donc la fonction COI du pronom réfléchi :

27. *Je me suis demandée : «Comment est-ce que je vais apprendre le français quand je suis entourée de la langue allemande?»

On peut toutefois remarquer que l'exemple (27) applique d'une certaine façon la règle simplifiée proposée par Gruaz (2012: 29), qui veut que tout PP de verbe pronominal s'accorde avec le sujet ${ }^{26}$.

Même si les résultats présentés ci-dessus s'avèrent globalement satisfaisants, la conclusion que l'on peut tirer de l'observation des erreurs commises en relation avec les verbes pronominaux est qu'à un niveau B1, la maitrise de l'analyse syntaxique, et

${ }^{26}$ Voir également Grevisse \& Goosse (1993) et Hanse (1987). 
particulièrement celle de la complémentation verbale, n’est pas assez développée pour permettre aux apprenants d'appliquer sans problème les règles complexes de l'accord du PP.

\subsection{Les participes passés au passif}

On pourrait penser que la voix passive, formée avec l'auxiliaire être, présente un taux de productions correctes proche de celle des verbes composés avec être. Or, il n'en est rien. En effet, si, pour les formes composées avec être, on trouvait un taux de formes correctes de $66,89 \%$, en moyenne, pour le passif, on observe un score nettement meilleur : 87,43\% (Tableau 8).

\begin{tabular}{|l|l|r|r|r|}
\hline Catégorie & Langues & \multicolumn{2}{|l|}{$\begin{array}{l}\text { Formes } \\
\text { correctes }\end{array}$} & \multicolumn{2}{|l|}{ Formes incorrectes } \\
\cline { 4 - 5 } & & 73,91 & Auxiliaire & \multicolumn{2}{|c|}{ Participe passé } \\
\hline \multirow{3}{*}{$\begin{array}{l}\text { ETRE / } \\
\text { PASSIF }\end{array}$} & anglais & 91,43 & 0,00 & 26,09 \\
\cline { 2 - 5 } & chinois & 90,77 & 0,00 & 8,57 \\
\cline { 2 - 5 } & espagnol & 81,08 & 5,41 & 9,23 \\
\cline { 2 - 5 } & kurde & 85,19 & 0,00 & 13,51 \\
\cline { 2 - 5 } & portugais & 90,91 & 0,00 & 14,81 \\
\cline { 2 - 5 } & russe & 87,43 & 0,57 & 9,09 \\
\hline Moyenne & & & & 12,00 \\
\hline
\end{tabular}

Tableau 8 : Pourcentage de réussite des PP au passif dans le corpus

\subsubsection{Formes correctes}

Sur les 350 formes au passif de notre corpus, 306 sont correctement réalisées, parmi lesquelles 104 sont non-marquées (masc. sing.) (ex. 28), soit une proportion nettement moins importante (34\%) que pour les verbes avec être (40,4\%). En d'autres termes, non seulement les formes passives sont globalement mieux réussies, mais encore elles présentent moins de formes pour lesquelles l'accord n'est pas visible, au masculin singulier. Même les formes plus difficiles à accorder correctement (féminin singulier, masculin et féminin pluriel) sont plus souvent correctes à la voix passive. 87 formes correctes sont au fém. sing. (29), 93 au masc. plur. (30) et 22 au fém. plur. (31) :

28. Ce sujet ne peut pas être traité d'une manière simpliste.

29. La liberté est conçue comme une capacité humaine [...]

30. Il y a toujours autant de couples mais de moins en moins sont mariés.

31. Certaines autres cultures ne sont pas encore informées par rapport à l'abolition de l'esclavage.

Les proportions des formes correctes / incorrectes selon le genre et le nombre s'avèrent intéressantes. On constate ainsi que le taux de réussite pour les formes masculines (masc. sing. 97,2\% ; masc. plur. 92,1\%) est plus élevé que pour les formes féminines (fém. sing. 78,4\% ; fém. plur. 81,5\%). Comme pour les auxiliaires avoir et être, c'est bien le genre qui provoque le plus de difficultés dans l'application des règles d'accord du PP. 


\subsubsection{Formes incorrectes}

À la différence des formes construites avec être, où les erreurs se répartissent entre auxiliaire mal choisi et PP mal accordé, les formes au passif ne présentent presque pas de problème quant à la sélection de l'auxiliaire. Cependant, on trouve deux cas incorrects, dans des productions de kurdophones (32 et 33). Il est à noter que le kurde utilise très peu le passif (Beddir Khan \& Lescot, 1970 : 198), et que celui-ci est construit avec l'auxiliaire hatin, qui signifie venir, et l'infinitif du verbe. Un apprenant kurdophone pourra donc hésiter entre être et avoir:

32. * La vie réfugié a déjà limité.

33. *Ta destinée a écrit déjà par la famille.

En ce qui concerne le deuxième type d'erreurs, celui qui porte sur le PP, dans 41 cas sur 42, c'est le PP qui n'est pas accordé. On retrouve ici les constatations faites pour l'auxiliaire être: la règle semble simple, mais son application est problématique, quoique dans une moindre mesure pour le passif. On trouve donc de nombreux cas comme les exemples (34) et (35):

34. *Les être humaine ont besoins de l'amour et d'être soigné par quelqu'un.

35. *Les patrons sont parfois aveuglé par leurs problèmes routinier.

Dans ces deux exemples, on constate que le phénomène de l'accord dans son ensemble n'est pas maitrisé, qu'il concerne le genre ou le nombre du nom (le substantif être n'est pas au pluriel), de l'adjectif ([être] bumaine, [problèmes] routinier) ou du PP. Ainsi, il semble bien que l'accord du PP à la voix passive subit exactement le même sort que celui des adjectifs. La comparaison avec cette catégorie grammaticale est d'autant plus pertinente que les proportions de productions correctes globales sont très proches, $90.39 \%$ pour les adjectifs attributs et $87,43 \%$ pour les passifs (voir Dubois, Kamber \& Skupien Dekens, 2014). Apparemment, les passifs sont perçus par les apprenants comme des adjectifs attributs, et non comme des formes verbales. Ceci peut expliquer la grande différence de réalisations correctes entre les formes avec l'auxiliaire être et les formes au passif.

Dans un seul cas, l'erreur est due à un accord fautif, en l'occurrence probablement avec le COD d'un autre verbe, précédant directement la forme passive :

36. Il n'aura aucune chance d'être soignée.

Au-delà des constatations faites ci-dessus sur les productions de formes passives par des apprenants allophones, c'est la grande fréquence de formes passives, justes ou fausses, qu'il convient de souligner. Sur les 1321 formes de notre corpus, 350 sont au passif, ce qui représente $26,5 \%$ du total. La fréquence de la voix passive dans un corpus de locuteurs natifs est beaucoup moins élevée, allant de 1\% à 15\% selon les genres textuels (Gaatone, 1998: 34). Même si on ne peut pas tenir ces chiffres établis sur des corpus restreints pour référence absolue, la différence est notable. Cette constatation est valable pour tous les groupes linguistiques représentés dans notre corpus, allant de $20 \%$ pour les anglophones à $29,6 \%$ pour les sinophones. Il semblerait que les apprenants préfèrent utiliser la voix passive plutôt que son 
équivalent actif et le font plutôt bien. S'agit-il d'une stratégie d'évitement consciente basée sur le sentiment que la voix passive se rapproche de l'usage de l'adjectif attribut avec le verbe être, perçu comme plus facile? Le fait que les enfants francophones semblent acquérir plus tardivement la voix passive (Gaatone, 1998: 35) tendrait à contredire cette hypothèse, même s'il est difficile de comparer une population d'adultes en cours d'apprentissage d'une L2 ou L3 à des enfants en cours d'acquisition de leur L1.

Finalement, en ce qui concerne la voix passive, on peut conclure qu'elle est surreprésentée dans notre corpus et réalisée nettement plus correctement que les formes composées avec l'auxiliaire être, alors que les règles d'accord sont les mêmes.

\section{Conclusion}

Contrairement aux dictées ou exercices lacunaires conçus spécifiquement par les enseignants pour tester l'application des règles de l'accord du participe passé, les textes d'étudiants utilisés dans ce corpus ne constituent pas un concentré de difficultés. Ils montrent l'usage concret des formes verbales composées par les apprenants et, partant, permettent d'établir une hiérarchie des besoins de ces derniers.

Notre étude sur corpus a permis de montrer que la principale difficulté, pour des scripteurs allophones de niveau B1, réside dans l'application de la règle la plus simple, à savoir l'accord du PP avec le sujet dans les formes composées avec l'auxiliaire être. Loin de constituer cet « ilot de sécurité » que les enseignants imaginent habituellement eu égard à la simplicité de la règle, cet accord est le principal problème rencontré par les apprenants de niveau B1. Il conviendrait donc impérativement de consacrer plus de temps aux formes composées avec l'auxiliaire être dans l'enseignement / apprentissage du FLE.

En ce qui concerne les PP avec avoir, on observe que le nombre de cas où le PP reste invarié correspond dans la très grande majorité des cas à une forme correcte $(90 \%)$. Les occurrences de constructions avec un COD placé avant sont rares, les apprenants n'utilisant que peu les relatives ou les pronoms objets dans leurs textes. Partant de ce constat, on pourrait sans dommage, dans un premier temps, s'en tenir à une règle simplifiée selon laquelle le PP ne s'accorde pas avec avoir. On peut imaginer que cette règle d'invariabilité éviterait aussi les accords fautifs avec le sujet que nous avons recensés dans le corpus. Ce n'est qu'une fois que ces règles seront acquises et correctement mises en pratique qu'on pourra introduire les principes d'accord avec le COD, puis les autres subtilités liées à l'accord du PP en français, tant qu'elles seront maintenues officiellement.

L'un des objectifs de cette étude était également d'observer les productions des étudiants à la lumière de leur L1. Certains résultats montrent, comme on pouvait s'y attendre, que le système de référence des apprenants joue un rôle dans leur compétence en L2. Si une telle démarche est difficile à mettre en œuvre dans une 
classe hétérogène sur le plan des L1, il n'en demeure pas moins qu'un enseignement efficace de l'accord du participe passé devrait tenir compte de ce paramètre et s'appuyer sur une approche contrastive mettant en lumière les similitudes et les différences des systèmes en présence. Ceci permettrait de prendre en compte les compétences plurilingues des apprenants à travers des activités grammaticales réflexives.

\section{Bibliographie}

Albert, C., Garnier, M., Rykner, A. \& SAint-Dizier, P. (2010), « Description et annotation des erreurs. Le cas des francophones s'exprimant en anglais », dans I. Biskri \& A. Jebali (éds.), Multilinguisme et traitement des langues naturelles, Québec, Presses de l'Université du Québec, p. 54-70.

Bedir Khan, E. D. \& Lescot, R. (1970), Grammaire Kurde (Dialecte Kurmandji), Paris, Adrien Maisonneuve.

BÉGUELIN, M.-J. (2002), «Faut-il simplifier les règles d'accord du participe passé ? », TRANEL, 37 , p. 163-179.

Berrendonner, A. (1983), Cours critique de grammaire générative, Lyon, Presses Universitaires de Lyon.

BRISSAUD, C. (1999), «La réalisation de l'accord du participe passé employé avec avoir. De l'influence de quelques variables linguistiques et sociales ", Langage et société, 88, p. 5-24.

Catach, N. (1989), Les délires de l'orthographe : en forme de dictionnaire, Paris, Plon.

CATACH, N., Duprez, D. \& LEGRIS, M. (1980), L'enseignement de l'orthographe : l'alphabet phonétique international, la typologie des fautes, la typologie des exercices, Paris, Nathan.

Dubois, M., KAmber, A. \& Skupien Dekens, C., (2014), «A quantitative and qualitative analysis of French L2 students' spelling problems: the case of adjective agreement », dans H. Tyne, V. André, C. Benzitoun, A. Boulton \& Y. Greub (éds.), French through Corpora: Ecological and Data-driven Perspectives in French Language Studies, Newcastle, Cambridge Scholars, p. 312-334.

FAYOL, M. (2007), «L'apprentissage de la morphologie du nombre», dans C. Brissaud et al. (éds), Nouvelles recherches en orthographe, Limoges, Editions Lambert Lucas, p. 119-135.

FrEI, H. (1929), La Grammaire des fautes, Bellegarde, Société anonyme des arts graphiques.

GaAtone, D. (1998), Le Passif en français, Paris / Bruxelles, Duculot.

GAlligani, S. \& BRUley, C. (2014), «De la notion d'interlangue à celle de compétence partielle et plurilingue : des exemples en FLE », Mélanges CRAPEL, 35, p. 31-45

Gervaix, P. (1995), Qu'avez-vous fait de votre participe passé?, Lausanne, Centre vaudois de recherches pédagogiques.

Granfeldt, J. \& Ågren, M. (2014), «De l'acquisition des langues à l'évaluation en FLE - le logiciel Direkt Profil en application », Mélanges CRAPEL, 35, p. 151-165. 
GRANGER, S. (2003), «Error-tagged learner corpora and CALL: a promising synergy », CALICO, 20/3, p. 465-480.

Grevisse, M. \& Goosse, A. (1993), Le Bon Usage, Bruxelles, de Boeck Duculot.

GRUAZ, C. (2012), Etudes pour une rationalisation de l'orthographe française, L'accord du participe passé (fasc. 4), Limoges, Lambert-Lucas.

HANSE, J. (1987), Nouveau dictionnaire des difficultés du français moderne, Paris / Bruxelles, Duculot.

KATTENBUSCH，D. (1993), "Interferenzbedingte Normverstösse beim Übersetzen in die Fremdsprache », dans R. Bauer et al. (éds.), Varietas delectat. Vermischte Beiträge zur Lust an romanischer Dialektologie ergänそt um Anmerkungen aus verwandten Disziplinen, Wilhelmsfeld, Egert.

LEROY, J. \& LEROY N. (1995), « La fréquence d'emploi des règles d'accord du participe passé », Enjeux, 34, p. 81-89.

LÜDELING, A. et al. (2005), "Multi-level error annotation in Learner Corpora », Corpus Linguistics 2005 Conference, Birmingham, article en ligne, www.corpus.bham.ac.uk/PCLC/FalkoCL2006.doc.

LuZZATI, D. (2011a), "Chapitre 8 : 'la matière graphique’», dans J.-C. Beacco, B. Blin, E. Houles, S. Lepage \& P. Riba (éds.), Niveau B1 pour le français : un référentiel, Paris, Didier.

LuZzATI, D. (2011b), «L'orthographe dans l'élaboration des niveaux de référence pour le français », TRANEL, 54, p. 9-20.

MATTHEY, M. (2013), «L'accord du participe passé en français langue première ou seconde : quelques données de terrain», dans S. Baddeley, F. Jejcic \& C. Martinez (éds), L'orthographe en quatre temps. 20e anniversaire des Rectifications orthographiques de 1990 : enseignement, recherche et réforme, quelles convergences? Paris, Champion, p. 105-118.

Nickel, G. \& NeHLS, D. (éds) (1982), Error Analysis and Second language learning, Heidelberg, Julius Groos.

PÉRY-WoODLEY, M.-P. (1995), "Quels corpus pour quels traitements automatiques ?», Traitement Automatique des Langues, 36/1-2, p. 213-232.

RAstelLi, S. \& Frontini, F. (2008), «SLA meets FLT research : the form/function split in the annotation of Learner Corpora ", Proceedings of TaLC 8, p. 446-451.

RAstelLI, S. (2009), « Learner Corpora without Error Tagging », Linguistik. Online, 38/2, p. 57-66.

Skupien Dekens, C., Kamber, A. \& Dubois, M. (2011), Manuel d'orthographe pour le français contemporain, Neuchâtel, Alphil - Presses Universitaires Suisses.

Teubert, W. (1998), «Korpus und Neologie», dans W. Teubert (éd.), Neologie und Korpus, Tubingue, Gunter Narr Verlag, p. 129-170.

Wilmet, M. (1999), Le Participe passé autrement, Paris / Bruxelles, Duculot. 